\title{
Muzzle Voltage Characteristics of Railguns
}

This paper was downloaded from TechRxiv (https://www.techrxiv.org).

\section{LICENSE}

CC BY 4.0

SUBMISSION DATE / POSTED DATE

$17-12-2021 / 21-12-2021$

CITATION

Tosun, Nail; Keysan, Ozan (2021): Muzzle Voltage Characteristics of Railguns. TechRxiv. Preprint. https://doi.org/10.36227/techrxiv.17211464.v1

$\mathrm{DOI}$

10.36227/techrxiv.17211464.v1 


\title{
Muzzle Voltage Characteristics of Railguns
}

\author{
Nail Tosun(D), Student Member, IEEE, Mustafa Karagöz, Member, IEEE, Ferhat Yurdakonar, Görkem Gülletutan, \\ and Ozan Keysan (Dember, IEEE
}

\begin{abstract}
Muzzle voltage is an essential diagnostic tool used in both contact resistance modeling and transition determination. However, it is challenging to stem the necessary meanings from the collected measurements. In this study, EMFY-3 launch experiments are used to model muzzle voltage characteristics to understand the transition mechanism better. These experiments have muzzle energies in the range between 1.69-2.85 $\mathrm{MJ}$ in ASELSAN Electromagnetic Launcher Laboratory. Six different launch tests with various rail current waveforms that ranged between 1.5-2.1 MA are used to investigate different scenarios. Some parameters which affect muzzle voltage are calculated with the 3-D Finite Element Method (FEM), i.e., rail mutual inductance $L_{m}$. Muzzle voltages are decomposed into subsections; each subsection is calculated with proper models. Simulation results are coherent with experimental measurements. Findings are compared with previous studies, and differences are explained with possible reasons. Even though we could not conclusively resolve which physical quantity starts to transition, the study showed that transition does not form a specific muzzle velocity, armature action integral, or down-slope rail current ratio.
\end{abstract} ing

Index Terms-railgun, muzzle voltage, electromagnetic model-

\section{INTRODUCTION}

$\mathbf{E}$ Lectromagnetic launchers (EMLs) are an accelerator that converts the reserved electrical energy to linear kinetic energy. It has two conducting rails, an armature, and a projectile. A pulsed-power supply (PPS) produces a pulse-shaped current in the order of a few MA in a slight duration (a few milliseconds) of time. Lorentz force acts on the armature, which is the underlying mechanism for propulsion.

At ASELSAN Inc., EMLs are explored in the naval context since 2014 [1]-[7]. Latterly, the EMFY-3 launcher is manifested with a $50 \times 75 \mathrm{~mm}$ rectangular bore and 6-m-length [8], [9]. The input energy of the PPS is doubled to $8 \mathrm{MJ}$, and the 2.91 MJ muzzle energy is obtained up to now. EMFY-3 is built in the ASELSAN Electromagnetic Launch Laboratory, which has an 8-MJ PPS system, a flash X-ray system, a 6-m-long catch tank, a Doppler radar system, and diagnostic tools [10]. The geometric parameters of the launcher are shared in Table I

One of the essential diagnostic tools for EML research is the muzzle voltage. The muzzle voltage of a railgun is the electrical potential difference between the muzzle ends of the rails. It is a valuable diagnostic instrument for railgun research because it can classify the electrical contact between armature and rails. As the contact can be formed in multiple phases,

N. Tosun, G. Gülletutan, and O. Keysan are with Middle East Technical University, Department of Electrical and Electronics Engineering, Ankara, Turkey. Email: keysan@metu.edu.tr

M. Karagöz, and F. Yurdakonar are with ASELSAN Inc., Ankara 06370 Turkey (e-mail: mkaragoz@aselsan.com.tr).

Corresponding Author: Ozan Keysan, keysan@metu.edu.tr
TABLE I

Geometric Parameters of the EMFY-3 Launcher.

\begin{tabular}{lc}
\hline Rail Height & $50 \mathrm{~mm}$ \\
Rail Separation & $75 \mathrm{~mm}$ \\
Rail Width & $50 \mathrm{~mm}$ \\
Rail Length & $6.1 \mathrm{~m}$ \\
\hline
\end{tabular}

i.e., solid, liquid metal-to-metal contact, or arcing contact, the muzzle voltage can be used to recognize transitions. Predetermining these transitions before launch and getting the rationalizations behind them is an imperative topic as these states are heavily associated with launch safety and efficiency.

In this paper, muzzle voltage characteristics are examined. For this regard, muzzle voltage decomposed into individual parts related to physical reasons. A 3-D Finite Element Method (FEM) model is used to calculate geometry-dependent parameters. Moreover, several hypotheses are constructed to possess mechanisms that form muzzle voltage. These are confronted with the previous studies in the literature, and empirical data originates from EMFY-3 experiments. Furthermore, the transition starting point is tried to correlate with some launch parameters, i.e., armature velocity and armature action integrals. At the end of the theoretical studies, a simulation model is constructed for the muzzle voltage. The organization of the paper is as follow; a broad literature survey about muzzle voltage is shared in Section II, the conducted hypotheses are explained with their validation in Section III, the simulation results are compared with the experimental data in Section IV, and the final thought are shared in Section V.

\section{LITERATURE SURVEY}

Muzzle voltage is an imperative diagnostic of a railgun. Sudden large jumps in the muzzle voltage line transitioning from a non-arcing, stable electrical contact to an arcing contact. Such transition can provoke energy losses as the contact resistance increase. Besides, material losses can occur due to the blow-off forces, or significant rail damages can happen during launches. These factors put a vital risk to the operation of the railgun. Apart from large transients, medium and slight amplitude variations also introduce information about the rail/armature interface condition [11]. Thus it is requisite to know the mechanism that creates muzzle voltage. The complete proof of all mechanisms is incomplete in the literature; investigations are proceeding. In this section, a brief literature survey is yielded in muzzle voltage research.

The muzzle voltage, denoted as $\mathrm{U}_{\mathrm{m}}$, is a measurement that consists of three contour integrals and an inductive term as in (1). These contours are illustrated in Fig. 1.b. Magnetic 


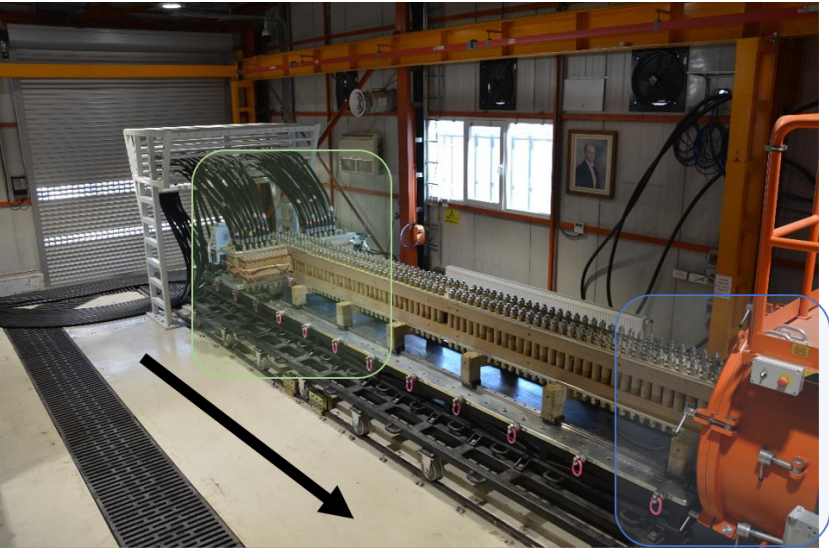

(a)

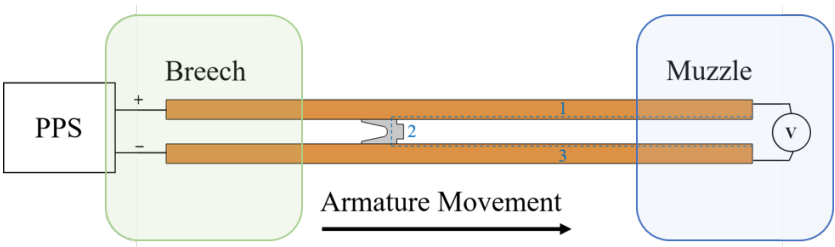

(b)

Fig. 1. EMFY-3 Launcher at the ASELSAN's Electromagnetic Launch Laboratory (a). Muzzle voltage measurement (b). Breech and muzzle points are illustrated with green and blue areas respectively.

flux ahead of the armature is the cause of the inductive term. Such a term can be interpreted as a mutual inductance since the current excitation is at the breech side. Such an analogy is illustrated in Fig. 2. Granting the armature is stationary, a railgun is a linear transformer whose secondary winding, $L_{2}$, is open-circuited. Thus, the mutual inductance, $M$, is equal to $L_{2}$. While the armature moves across the rails, breech inductance, referred to as primary inductance $L_{1}$, is increased. Nevertheless, $L_{2}$ is still constant in most of the launch time as the magnetic field ahead of the armature, which constitutes $L_{2}$, is much weaker than its behind. Magnetic field density vectors around armature are illustrated in Fig. 3. Then (1) can be arranged as (2), where $I_{r}$ is the rail current, and $R_{c}$ is the armature resistance in addition to contact resistance. This sum is denoted as $R_{c}$ throughout the paper for the sake of simplicity.
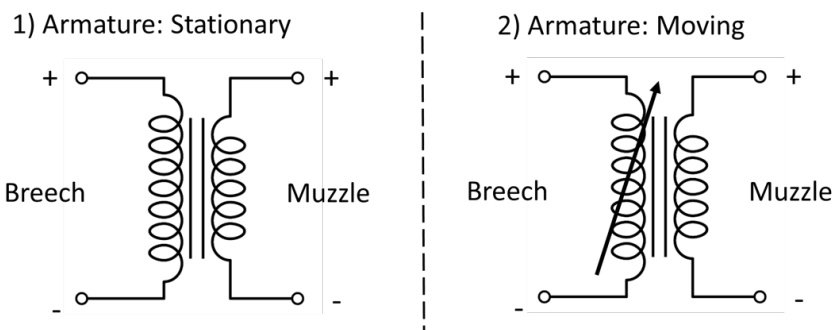

Fig. 2. The transformer analogy.

$$
\begin{gathered}
U_{m}=\oint_{C_{1}} \vec{E}_{1} d \vec{l}+\oint_{C_{2}} \vec{E}_{2} d \vec{l}+\oint_{C_{3}} \vec{E}_{3} d \vec{l}+\frac{d \Phi}{d t} \\
U_{m}=I_{r} R_{c}+L_{m} \frac{d I_{r}}{d t}
\end{gathered}
$$

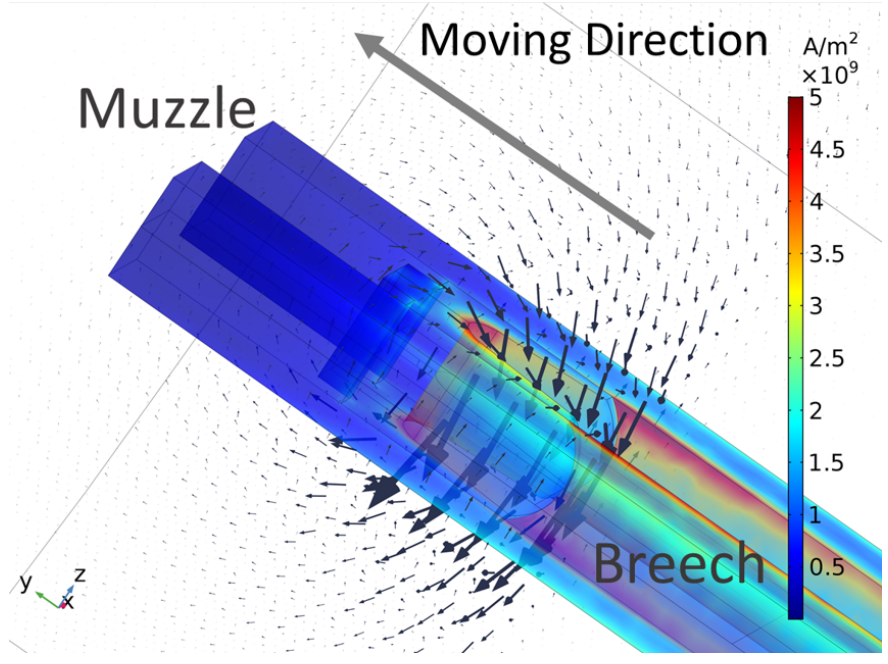

Fig. 3. The magnetic field density vectors around armature are illustrated with black arrows. Current density values are showed with colors. Muzzle and breech sides are indicated. Armature moving direction is illustrated with a gray arrow.

(2) is a prevalent insight when the muzzle voltage is decomposed considering the transformer analogy. Dreizin and Barber contribute another term that is closely associated with armature velocity [12]. As the armature move, a new rail portion is experienced with a magnetic field gradient. Such gradients induce a current in the inner surfaces of the rails (paths 1, and 3 from Fig. 1.b.). This contribution becomes dominant as velocity rises. The term denoted as $\mathrm{U}_{\mathrm{r}}$ is expressed analytically and demonstrated in (3), where $\mathrm{k}$ is a geometry-dependent parameter, $\rho_{\mathrm{r}}$ is the rail resistivity, $\mathrm{V}$ is the armature velocity, $\mathrm{s}$ is the rail separation. With the extension of $\mathrm{U}_{\mathrm{r}}$ the muzzle voltage becomes (4).

$$
\begin{gathered}
U_{r}=k I_{r} \sqrt{\mu_{0} \rho_{r} V / s} \\
\bar{U}_{m}=I_{r} R_{a}+L_{m} \frac{d I_{r}}{d t}+k I_{r} \sqrt{\mu_{0} \rho_{r} V / s}
\end{gathered}
$$

The content of the $R_{c}$ is an essential topic, as Dreizin stated. $\mathrm{C}_{2}$ evaluates both armature and contact resistances when the contact is considered homogeneous. However, when the armature exceeds a few hundred $\mathrm{m} / \mathrm{s}$, the current distribution is skewed into its tails. This phenomenon is known as the velocity skin effect (VSE). The current distributions where the armature is stationary and moving are illustrated in Fig. 4 to demonstrate its influence. As the armature moves, it is not clear that $\mathrm{C}_{2}$ will take into account contact resistance. The reason is that the current pass through $\mathrm{C}_{2}$ is significantly diminished as VSE becomes dominant. Even if magnetic field gradients in the rail portion ahead of the armature can create significant eddy currents, it is unclear to these currents can 
form a potential difference that can totally measurable through line contours.

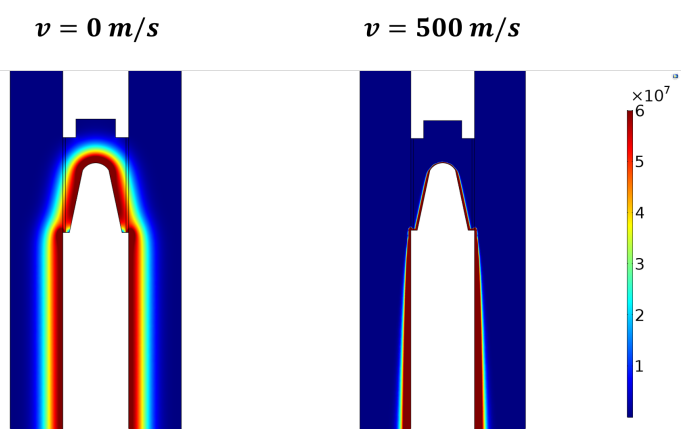

Fig. 4. 2-D transient FEM results with stationary and moving armature. VSE narrows the current distribution which may affect the muzzle voltage.

Experimental proof of the $\mathrm{U}_{\mathrm{r}}$ is made by Parker [13]. Therefore, (4) seems a good way to examine the muzzle voltage, excluding one problem. As Parker stated, the value of the $\mathrm{k}$ is critical, and the analytical formulation has not adequate accuracy. A 3-D FEM model could be used to evaluate $\mathrm{k}$, whereas it would have a significant computational load. The objective is to examine induced currents due to the VSE in $3-\mathrm{D}$, which is still a challenging computational problem.

Chen et. al added a motional electromotive force (EMF) term to the $\mathrm{U}_{\mathrm{m}}[14]$. This term is denoted as $U_{e}$, which is given in (5). However, there are mismatches between simulations and experiments. However, they conclude that the mismatches occurred due to as $\mathrm{U}_{\mathrm{e}}$ is added to $\mathrm{U}_{\mathrm{m}}$ as a whole. The term should be multiplied with a proportionality constant as $C_{2}$ does not include the whole contact.

$$
U_{e}=\frac{1}{2} L^{\prime} v I_{r}
$$

Unlike Chen et. al, we consider that there should not be any motional EMF component in the $\mathrm{U}_{\mathrm{m}}$ as there is in the breech voltage. When the armature, a conductor, moves in its magnetic field, it creates an EMF. This can be explained by a voltage caused by increased inductance as in the first term in (6), phenomenologically. However, it is crucial that where the EMF is built up in the railgun. The conductor movement in the magnetic field creates an illusion that the EMF built up at the armature as a contact voltage. However, as the armature is connected to rails electrically, the EMF is created in the railgun as a whole. For that reason, breech voltage measurements have a clear slope correlated with armature acceleration. The EMF discussions are mentioned in [4], interested readers can find more detail in that article.

$$
\mathcal{E}=\frac{d \lambda(t)}{d t}=\frac{d L(t)}{d t} I(t)+L(t) \frac{d I(t)}{d t}
$$

Although series of works were conducted on the muzzle voltage mechanisms, there are still missing points to fully emulate it. In the following section, several hypotheses are constructed to understand more about muzzle voltage. These hypotheses are tested with various experiments to build a simulation model.

\section{HYPOTHESES}

In the previous section, several muzzle voltage formulations obtained in the literature are exhibited. Although some contributions are solid, a few of them contradict themselves. In this section, the primary objective is to hypothesize some of them to achieve a better model. A typical muzzle voltage waveform is illustrated in Fig. 10 with its rail current, $I_{\text {rail }}$, and armature velocity, $v$, curves. Three separate regions are defined, each of them illustrated with a distinct color. $\mathcal{R}_{1}$ represents the first region where $I_{\text {rail }}$ is continuously rising; however, $v$ is little. In $\mathcal{R}_{2}, I_{\text {rail }}$ is constant, as a pulse-shape rail current is wanted. Since $\dot{I}_{\text {rail }}$ is retained minimal, there is not a significant inductive voltage component in the muzzle voltage. In $\mathcal{R}_{3}, I_{\text {rail }}$ is decreasing whereas muzzle voltage increases rapidly and ends its peak at the armature exit time. Conceding three regions, five hypotheses are built and examined.

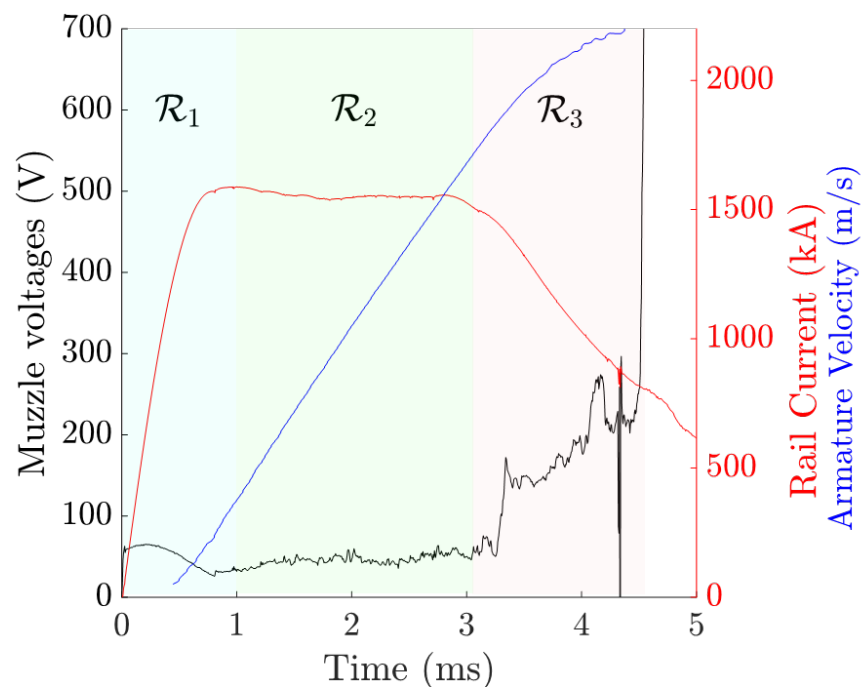

Fig. 5. Typical muzzle voltage with its rail current and armature velocity curve which is obtained with a Doppler radar. Each region of interest is illustrated with different colors.

The first hypothesis is constructed as following;

Hypothesis $1\left(\mathcal{H}_{1}\right)$ : The muzzle voltage contribution in the $\mathcal{R}_{1}$, consists of an inductive component due to $L_{m}$, and resistive component due to $R_{\text {contact }} . L_{m}$ is constant, whereas $R_{\text {contact }}$ has transients.

$\mathcal{H}_{1}$ is rooted in the transformer analogy where the armature can be pretended stationary. Thus, (2) is applied to calculate $U_{m}$. However, it is still important to determine how much voltage induced by $L_{m}$ and $R_{\text {contact }}$ respectively to understand whether one of two dominant or not. In Fig. 6 rail current measurements and corresponding velocity curves are illustrated. $\mathcal{R}_{1}$ is indicated with turquoise. Each rail current has distinct $\hat{I}_{\text {rail }}$; thus $\dot{I}_{\text {rail }}$ values are various at $\mathcal{R}_{1}$. Therefore, the inductive component of the muzzle voltage can be differentiated for each launch. It is clear that the armature has little motion, so there is no reason for a variance in $L_{m}$. Muzzle voltages at the $\mathcal{R}_{1}$ are demonstrated in Fig. 77. An increase in muzzle voltages with a subsequent decrease is observed in both measurements. It should be noted that, in 


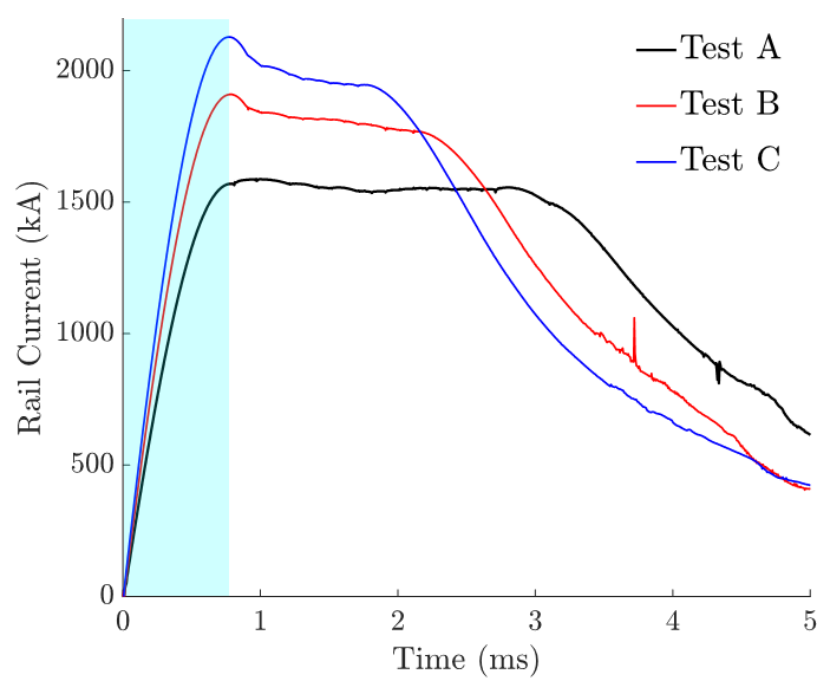

(a)

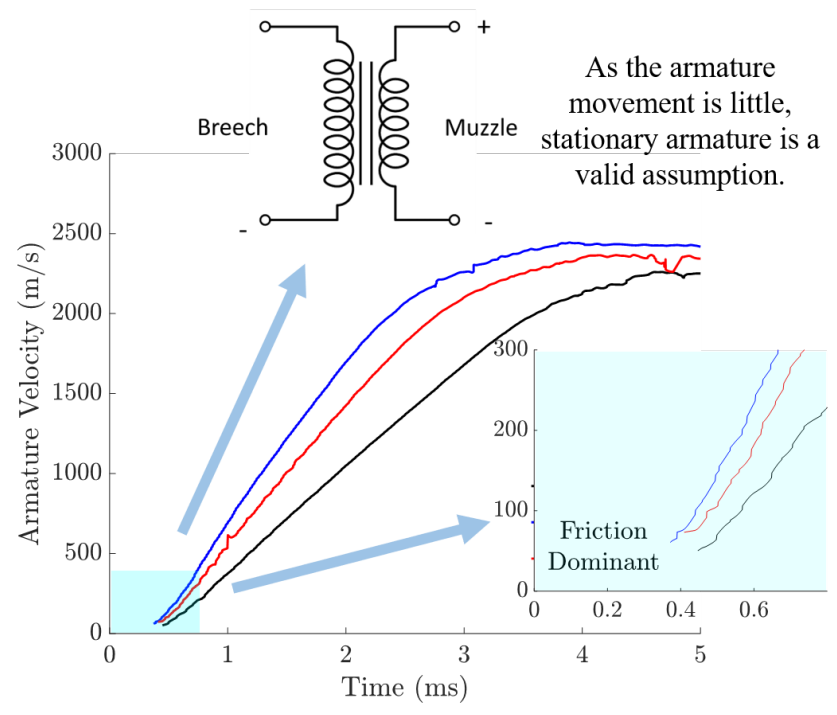

(b)

Fig. 6. Rail current measurements at different tests (a). $\mathcal{R}_{1}$ is illustrated with turquoise. Each test has dissimilar peak rail current, $\hat{I}_{\text {rail }}$, with identical current rise time, $t_{r}$, thus; their $\dot{I}_{r a i l}$ is differ. Corresponding velocity curves are shown in (b). As the friction is dominant at $\mathcal{R}_{1}$, stationary armature is a valid assumption.

$\mathcal{R}_{1}, I_{\text {rail }}$ is increasing whereas $\hat{I}_{\text {rail }}$ declines continuously. Therefore, if the inductive component is solely dominant, muzzle voltages should have a continuous decreasing trend. As $L_{m}$ is considered as constant, $R_{\text {contact }}$ should have a dynamic value. Conveniently, $R_{\text {contact }}$ rises concerning its initial value, as the electrical contact is heated with $I_{\text {rail }}$ and friction. The exact variations of $R_{\text {contact }}$ are difficult to calculate, although they may be extracted from empirical data. The inductive component can be calculated, as the $L_{m}$ is exploited with 3-D FEA. However, $\mathcal{H}_{1}$ is valid; $R_{\text {contact }}$ must have significant dynamics that can influence muzzle voltage. Both inductive and resistive components are critical and should be calculated when $\mathcal{R}_{1}$ is modeled.

The second hypothesis is constructed as following;

Hypothesis $2\left(\mathcal{H}_{2}\right)$ : Muzzle voltage is not depend on the

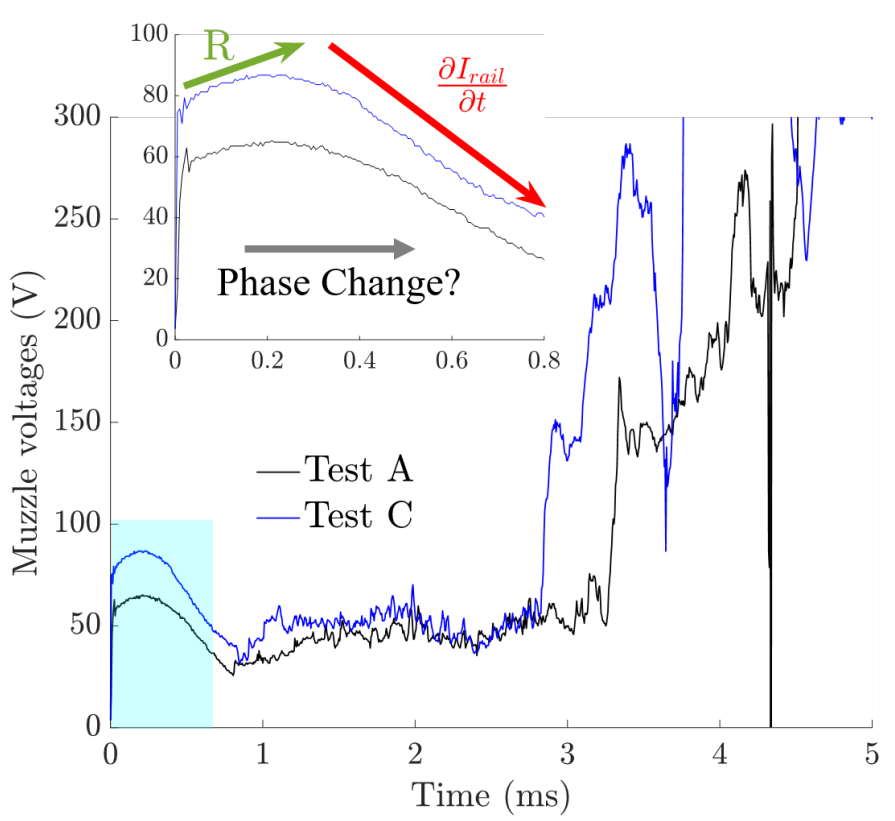

Fig. 7. Muzzle voltages at $\mathcal{R}_{1}$. Slight increase with subsequent decrease is observed which indicates multiple transient mechanisms are occurred. As $\dot{I}_{\text {rail }}$ is monotonously decreasing, transients occur due to resistive components.

module's capacitor voltage, $V_{c}$, at $\mathcal{R}_{1}$ and $\mathcal{R}_{2}$.

To confirm these claims, $\mathcal{R}_{2}$ should be investigated. The starting point of the study is $\mathcal{H}_{2}$. In Fig. 8, rail currents with comparable $\hat{I}_{\text {rail }}$, diverse pulse width and $V_{c}$, are shown. $\mathcal{R}_{2}$ is colored to designate the region. It should be noted that throughout $\mathcal{R}_{2}$, all test has comparable armature action integrals, and $v$ 's, as equally weighted armatures, are used; the only distinction is $V_{c}$. However, muzzle voltages are the same, excluding inductive spikes due to PPS firings. Thus muzzle voltage is invariant to $V_{c}$. This is convenient as the EML is deemed a current-driven device. Therefore, $\mathcal{H}_{2}$ is confirmed.

The third hypothesis is constructed as following;

Hypothesis $3\left(\mathcal{H}_{3}\right)$ : At $\mathcal{R}_{2}, R_{\text {contact }}$ is constant as long as the sliding contact has stable liquid film.

In $\mathcal{R}_{2}, \dot{I}_{\text {rail }}$ is vanished to zero to create pulse shape current; thus, the inductive voltage is minimal. From Fig. 8)(b), it is clear that muzzle voltage is steady. Thus, $R_{\text {contact }}$ can be considered as constant. It should be noted that $\mathcal{H}_{2}$ focuses $\mathcal{R}_{2}$, as the friction lose effect before $\mathcal{R}_{2}$, which indicates that the liquid form is received at the electrical contact. However, someone can claim that the value of $R_{\text {contact }}$ may differ with different $I_{\text {rail }}$ excitations; as Fig. 8 shows almost identical excitations throughout $\mathcal{R}_{1}$, and $\mathcal{R}_{2}$. For that reason, experiments with different $\hat{I}_{\text {rail }}$ should be considered. In Fig. 9 (a) an experiment with a different $\hat{I}_{\text {rail }}$ is added. Muzzle voltages in $\mathcal{R}_{2}$ are demonstrated only; actual values are dashed, whereas the moving-averaged values are shown with bold lines. In Fig. 9 (b), $R_{\text {contact }}$ distributions at $\mathrm{R}_{2}$ are illustrated with a BoxWhisker plot. Legend 1 to 3 indicate experiments with $\hat{I}_{\text {rail }}$ is $1.5 \mathrm{MA}$ whereas, legend 4 indicated the experiment with $\hat{I}_{\text {rail }}$ is 2 MA. Red dots in Fig. 9 are outliers that occur due to inductive voltage spikes related to switching PPS modules. 


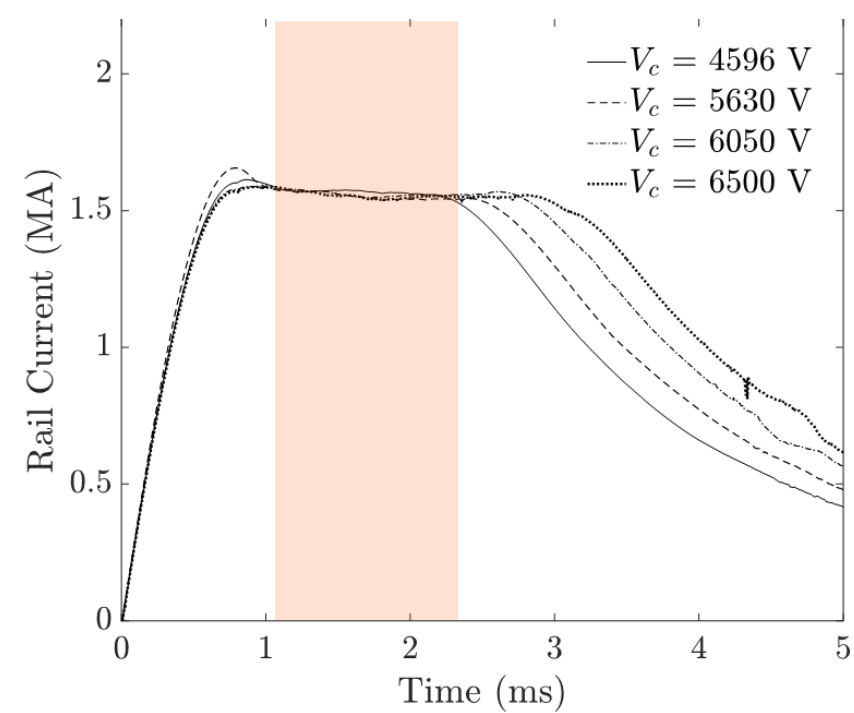

(a)

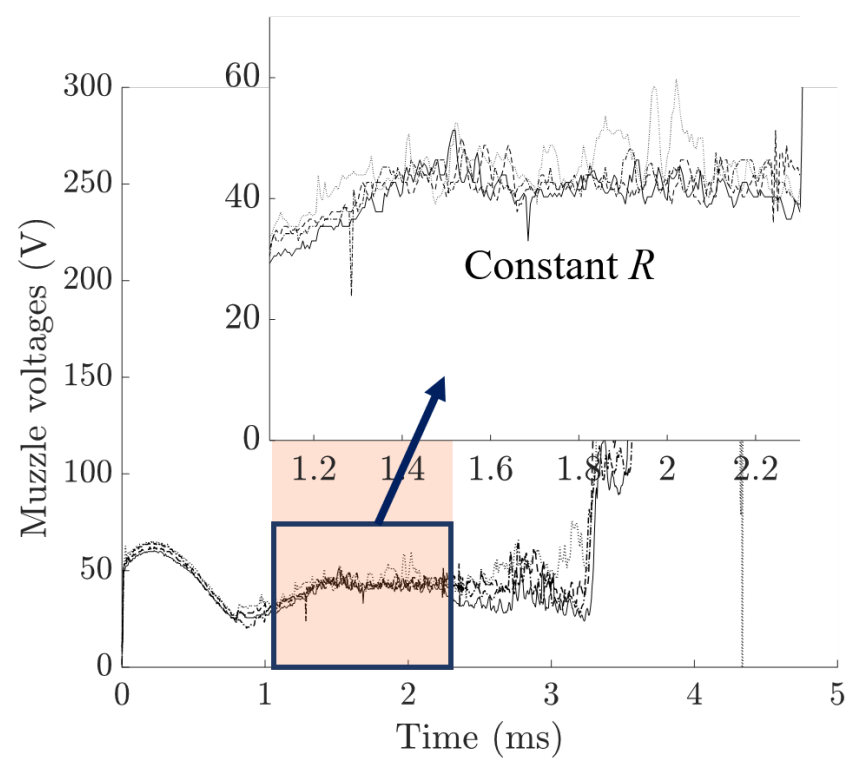

(b)

Fig. 8. Rail current measurements with different pulse-width, and $V_{c}$ (a). Corresponding muzzle voltages (b). $\mathcal{R}_{2}$ is colored in both subfigures. It should be noted that $I_{\text {rail }}$ is vanished to zero in $\mathcal{R}_{2}$ which means there is no inductive component at muzzle voltages.

$R_{\text {contact }}$ is deemed to constant around 25-30 $\mu \Omega$, regardless of $I_{\text {rail }}$ which verify $\mathcal{H}_{3}$. In Fig. 9 (b), the legend 4 is fatter than others, even though the median is close. This can be explained with Test A's $I_{\text {rail }}$ in Fig. 6. (a). Test A's $I_{\text {rail }}$ is not flat as experiments with 1.5 MA $I_{\text {rail }}$ s (demonstrated in Fig. 8. (a)), which extract some inductive components to muzzle voltage at $\mathcal{R}_{2}$.

Hypothesis $4\left(\mathcal{H}_{4}\right)$ : Muzzle voltage is not depend on the armature velocity at $\mathcal{R}_{1}$ and $\mathcal{R}_{2}$.

$\mathcal{H}_{4}$ is a strong argument respecting previous studies that verify its contrast [13]. However, in $\mathcal{R}_{2}$, there is not any muzzle voltage deviation even though $v$ exceeds $1500 \mathrm{~m} / \mathrm{s}$. Our conclusion from the contradiction with [13] is the follow-

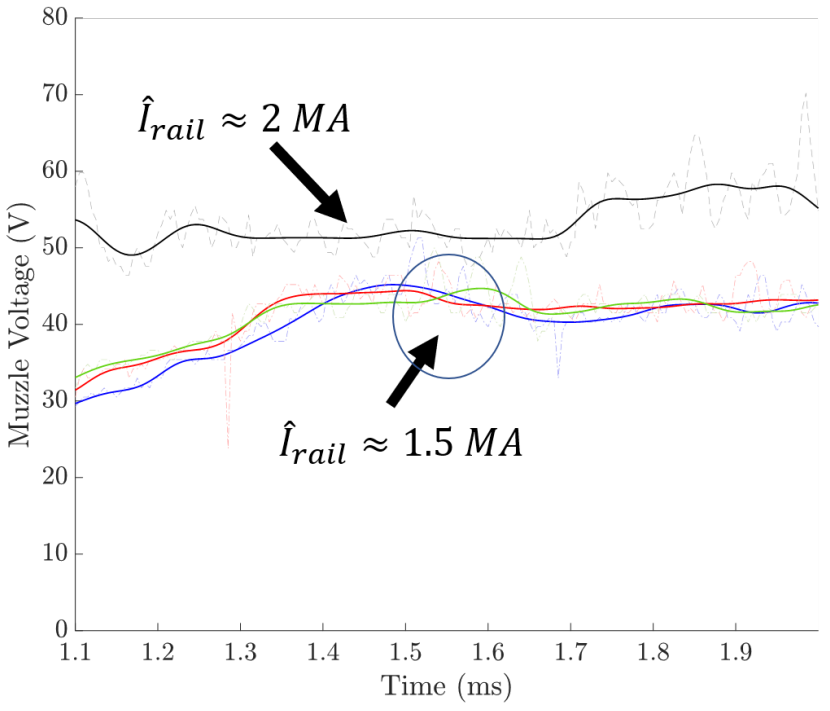

(a)

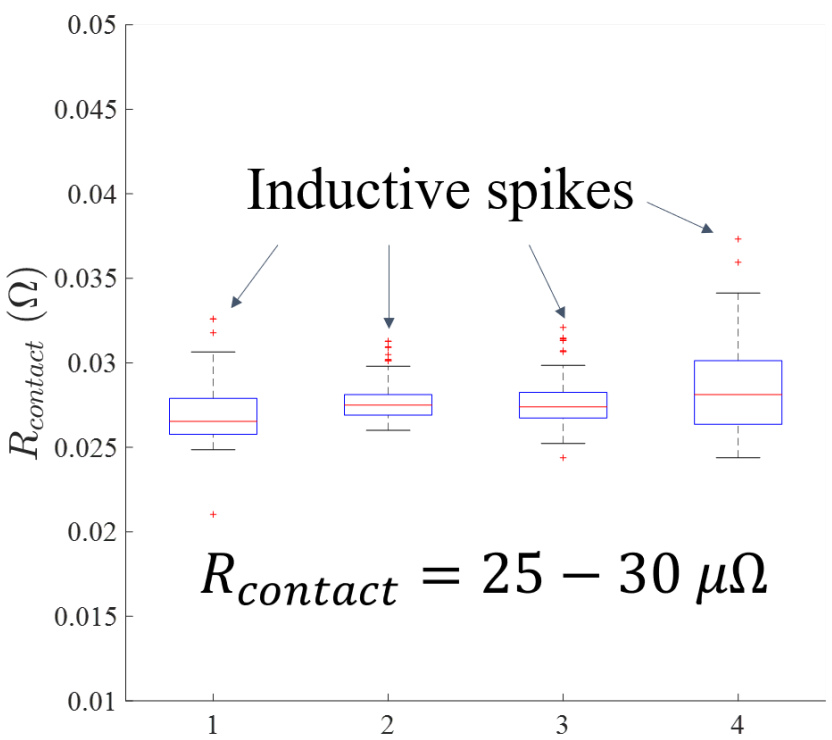

(b)

Fig. 9. Muzzle voltage measurements at $\mathcal{R}_{2}$ (a). Their $\hat{I}_{\text {rail }}$ are demonstrated. Actual values are dashed, whereas moving-average filtered values are illustrated with bold lines. $R_{\text {contact }}$ values at $\mathcal{R}_{2}$ are visualized with BoxWhisker plot (b).

ing; the EMFY-3 launcher has non-conductive containment, whereas in [13] the containment is laminated steel which can amplify magnetic field gradients ahead of the armature. Such amplification increases the muzzle voltage content of this phenomenon such that it is observable, unlike our empirical data.

Hypothesis $5\left(\mathcal{H}_{5}\right)$ : The moment that transition starts is not dependent solely armature action integral, muzzle current ratio, and armature velocity independently.

In $\mathcal{R}_{3}$, muzzle voltages have significant jumps, although $I_{\text {rail }}$ are continuously decreasing. Such increment can be associated with an increase in $R_{\text {contact }}$ as negative $\dot{I}_{\text {rail }}$ creates separative forces between rail and armature. Moreover, 
$R_{\text {contact }}$ is dependent on contact pressure which is linked with $I_{\text {rail }}$. However, these muzzle voltage increments are not steady; they seem like random high transients. Thus, they point out unhealthy, unstable arcing electrical contact. It is vital to determine when and why the transition occurs.

To correlate the transition moment with launch parameters, the first candidate armature action integral is used. The armature action integral (AAI) is the time integral of the $I_{\text {rail }}^{2}$ as demonstrated in (7), where $t_{\text {exit }}$ is the armature exit time. As AAI is a cumulative term that reflects the armature's electrical load, it may be associated with the transition.

$$
A A I=\int_{0}^{t_{\text {exit }}} I_{\text {rail }}^{2} d t
$$

In Fig. 10, AAI values with respect to time are illustrated with different launches. The transition moments are indicated with vertical dashed lines and arrows. These measurements have rail currents whose demonstrated in Fig. 8.a. All of them have the same $\hat{I}_{\text {rail }}$, which indicates launches have the same linear current density, similar accelerations, and identical armatures. However, transitions occured at different AAI values. Fig. 10 also shows that armature velocity can not be a candidate also. Transitions occurred between 1635-1820 $\mathrm{m} / \mathrm{s}$, which is a large velocity span. Again, the linear current density is set constant, and identical armatures are used in these experiments.
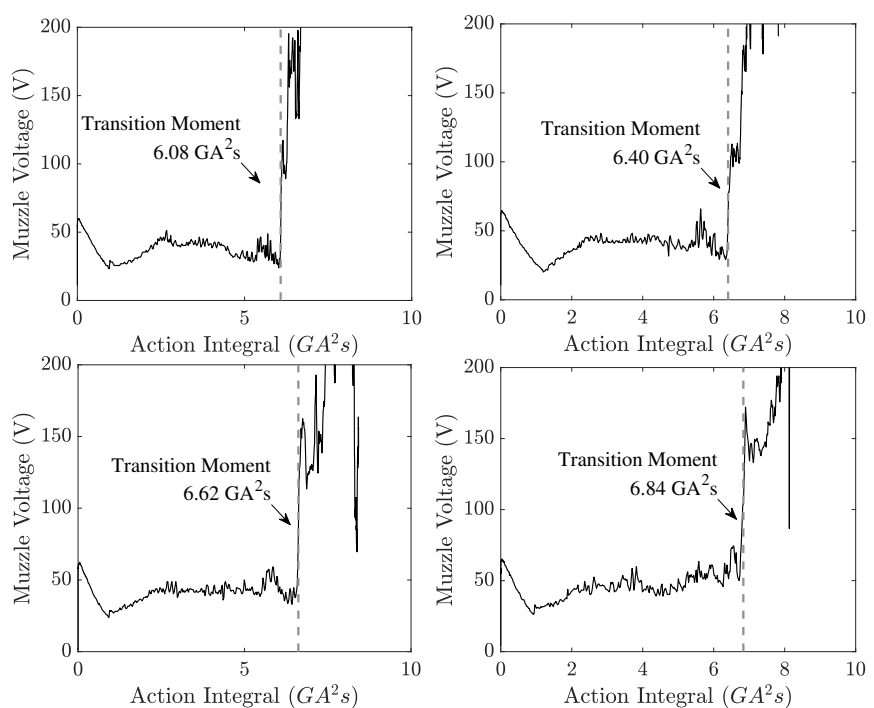

Fig. 10. Muzzle voltages with respect to armature action integrals.

Down-slope $I_{\text {rail }}$ ratio is associated with transition first by Satapaty et. al. [15]. A statistical analysis of existing data showed that contact transitions happen at approximately $80 \%$ of $\hat{I}_{\text {rail }}$ during down-slope. In the EMFY-3 experiment, the transition occurred 93\%-61\% down-slope $I_{\text {rail }}$ ratio without demonstrated any trend solely. Our conclusion as the transition can not be modeled with launch parameters, $\mathcal{R}_{3}$ can not be simulated accurately.

\section{REsults}

In this section, the simulation model, which is obtained by considering $\mathcal{H}_{1}-\mathcal{H}_{5}$, compared with experimental results.

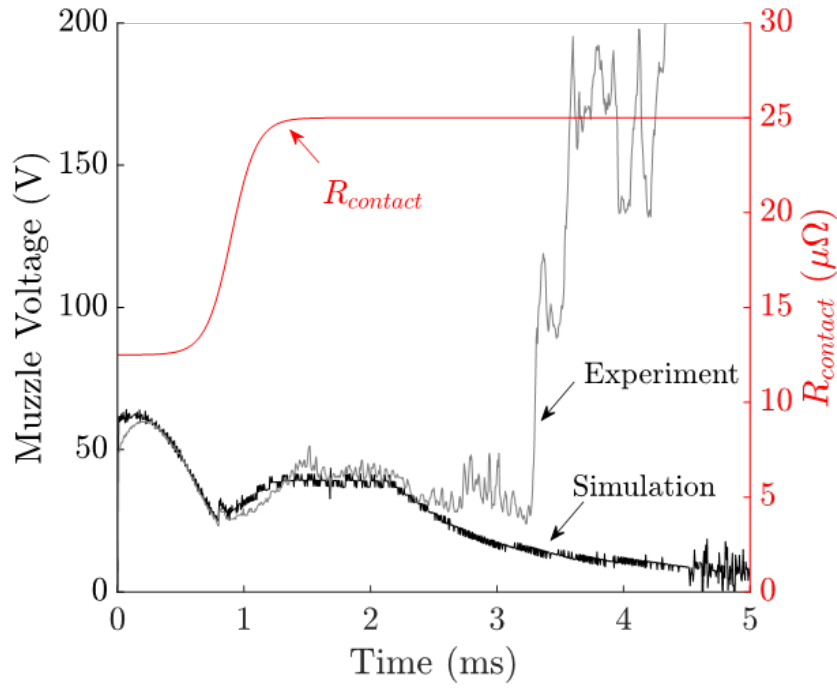

(a)

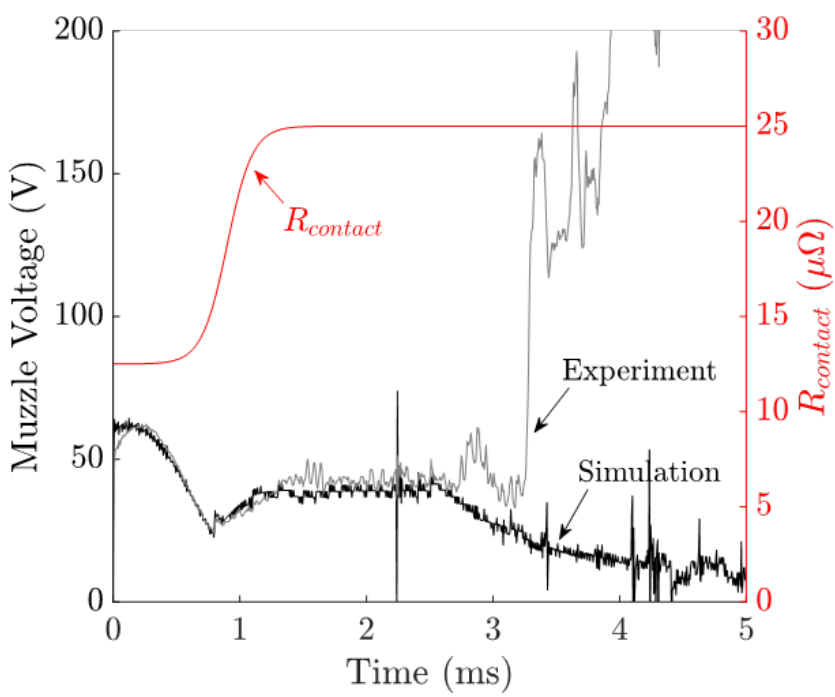

(b)

Fig. 11. Simulation and experimental muzzle voltage waveforms are illustrated with $R_{\text {contact }}$.

The model is built with (2), as $\mathcal{H}_{4}$ states that muzzle voltage is independent of $v$. As aforementioned $L_{m}$ is a geometrydependent parameter, it is exploited with 3-D FEA with fluxcounting method [4]. $L_{m}$ is $18 n H$ for the EMFY-3 launcher. $R_{\text {contact }}$ is constant around 25-30 $\mu \Omega$ at $\mathcal{R}_{2}$, however, it has a dynamic value in $\mathcal{R}_{1}$ as $\mathcal{H}_{1}$ indicates. Even if the dynamic nature of the $R_{\text {contact }}$ seems challenging to include a mathematical model, a simple sigmoid function is used. The sigmoid function is given in (9) where $\gamma$, and $\zeta$ determine the transition velocity, and position respectively.

$$
\sigma(\gamma, \zeta, t)=\frac{1}{1+e^{-\gamma(t-\zeta)}}
$$

$\gamma$, and $\zeta$ are not calculated analytically, they extracted through muzzle voltage values considering (2). $\gamma$ is equal to 10 , and $\zeta$ is equal to $0.910^{-3}$ give consistent results with experimental findings. 
Simulated and measured muzzle voltages are illustrated with the dynamic $R_{\text {contact }}$ in Fig. 11. The model fit muzzle voltages without any discrepancy in $\mathcal{R}_{1}$ and $\mathcal{R}_{2}$. The model starts to deviate when $I_{\text {rail }}$ begins decreasing. However, this is expected as the $I_{\text {rail }}$ becomes less, the contact pressure also diminishes, which eventually increases $R_{\text {contact }}$. Such a mechanism is a lack in the model. Moreover, the complete transition region is also excluded in the model as aforementioned in $\mathcal{H}_{5}$ explanation. These regions are demonstrated in Fig 12.

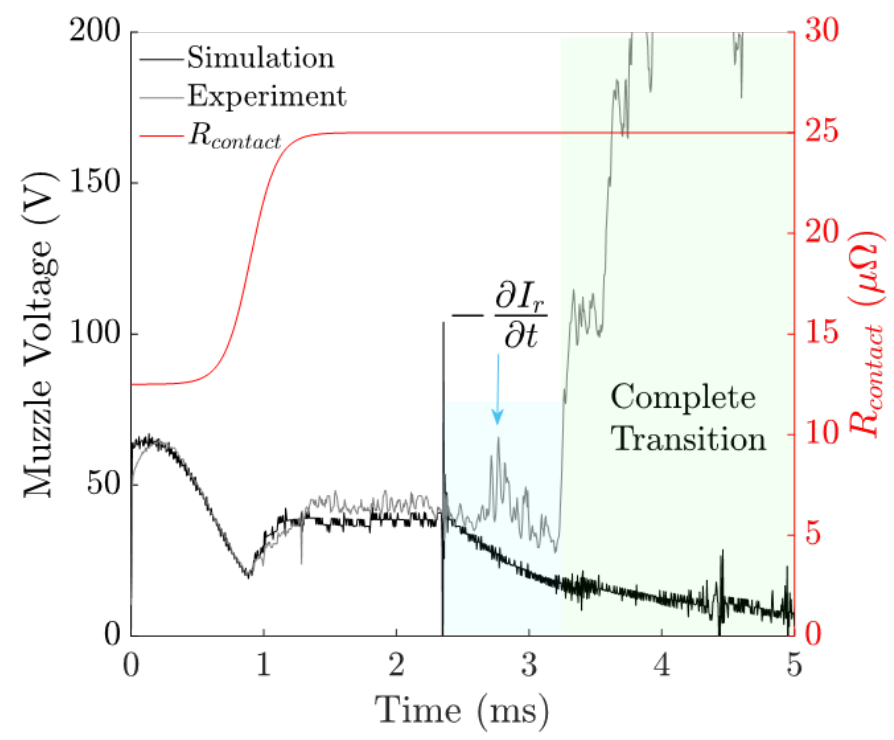

Fig. 12. $\mathcal{R}_{3}$ and the complete transition zone.

\section{CONCLUSION}

In this study, several hypotheses on muzzle voltage mechanisms are investigated with empirical findings. These are compared with previous studies in the literature. Moreover, a simulation model is proposed to model muzzle voltage at pretransition regions. The remarks which are obtained throughout the survey are listed as:

1) The muzzle voltage contribution in the $\mathcal{R}_{1}$, has an inductive component due to $L_{m}$, and resistive component due to $R_{\text {contact }} . L_{m}$ is steady, whereas $R_{\text {contact }}$ includes transients. $R_{\text {contact }}$ can be modeled as a simple sigmoid function which emulate the phase change.

2) Muzzle voltage is not depend on the module's capacitor voltage, $V_{c}$, as the EML considered a current-driven device.

3) At $\mathcal{R}_{2}, R_{\text {contact }}$ is constant $(25-30 \mu \Omega)$ as the sliding contact has stable liquid film. $R_{\text {contact }}$ is invariant from $I_{\text {rail }}$ as long as the contact is stable.
4) Muzzle voltage is not depend on the armature velocity at $\mathcal{R}_{1}$ and $\mathcal{R}_{2}$.

5) The moment that transition starts is not solely dependent on armature action integral, muzzle current ratio, and armature velocity alone.

\section{ACKNOWLEDGMENT}

The authors would like to thank the other ASELSAN Team members whose support made this article possible.

\section{REFERENCES}

[1] E. Durna, Y. Çevik, M. Karagöz, and A. Civil, "Design and implementation of a hierarchical control system architecture for a modular pulsed power supply system," in 2016 IEEE International Power Modulator and High Voltage Conference (IPMHVC), 2016, pp. 594-597.

[2] A. Civil, Cavbozar, M. Karagöz, E. Tan, and Y. Çevik, "Design and improvement of a pulse shaping inductor for a pulsed power system," in 2017 IEEE 21st International Conference on Pulsed Power (PPC), 2017, pp. 1-3.

[3] M. Karagoz, Y. Çevik, E. Tan, A. Civil, O. Cavbozar, U. Gocmen, B. Yildirim, E. Durna, and M. Sahin, "Aselsan emfy-1 electromagnetic launcher: First experiments," in 2017 IEEE 21st International Conference on Pulsed Power (PPC), 2017, pp. 1-3.

[4] N. Tosun, H. Polat, D. Ceylan, M. Karagoz, B. Yıldırım, Güngen, and O. Keysan, "A hybrid simulation model for electromagnetic launchers including the transient inductance and electromotive force," IEEE Transactions on Plasma Science, vol. 48, no. 9, pp. 3220-3228, 2020.

[5] D. Ceylan, M. Karagöz, Y. Çevik, B. Yıldırım, H. Polat, and O. Keysan, "Simulations and experiments of emfy-1 electromagnetic launcher," IEEE Transactions on Plasma Science, vol. 47, no. 7, pp. 3336-3343, 2019.

[6] N. Tosun, D. Ceylan, H. Polat, and O. Keysan, "A comparison of velocity skin effect modeling with 2-d transient and 3-d quasi-transient finite element methods," IEEE Transactions on Plasma Science, vol. 49, no. 4 pp. $1500-1507,2021$

[7] M. Karagoz, A. Civil, B. Yildirim, E. B. Yurdakul, E. Durna, E. Tan, O. Cavbozar, U. Gocmen, and Y. Cevik, "Aselsan electromagnetic launch laboratory: First shot," IEEE Transactions on Plasma Science, vol. 48, no. 4, pp. 802-807, 2020

[8] N. Tosun et al., "Inductance gradient calculations of emfy-3 electromagnetic launcher," IEEE Transactions on Plasma Science, 2021, submitted for publication, under review.

[9] N. Tosun et al., "Bus impact on the inductance distribution of electromagnetic launchers," IEEE Transactions on Plasma Science, 2021, submitted for publication, under review.

[10] M. Karagoz, A. Civil, B. Yildirim, E. B. Yurdakul, E. Durna, E. Tan, O. Cavbozar, U. Gocmen, and Y. Cevik, "Aselsan electromagnetic launch laboratory: First shot," IEEE Transactions on Plasma Science, vol. 48, no. 4, pp. 802-807, 2020.

[11] D. Melton and F. Stefani, "Noise component in muzzle voltage traces," IEEE Transactions on Magnetics, vol. 41, no. 1, pp. 214-219, 2005.

[12] Y. Dreizin and J. Barber, "On the origins of muzzle voltage," IEEE Transactions on Magnetics, vol. 31, no. 1, pp. 582-586, 1995.

[13] J. Parker, "Experimental observation of the rail resistance contribution to muzzle voltage [in railguns]," IEEE Transactions on Magnetics, vol. 35, no. 1, pp. 437-441, 1999.

[14] L. Chen, J. He, Y. Pan, and Z. Xiao, "Muzzle voltage of railgun in zero velocity and launch experiments," in 2008 14th Symposium on Electromagnetic Launch Technology, 2008, pp. 1-5.

[15] S. Satapathy and H. Vanicek, "Down-slope contact transition in railguns," IEEE Transactions on Magnetics, vol. 43, no. 1, pp. 402-407, 2007. 